\title{
A Formula of Newton
}

\author{
Eugene F. Krause
}

Department of Mathematics, The University of Michigan, Ann Arbor, Michigan 48104

\section{PuRPose}

The purpose of this paper is to revive an old formula of Newton which is well known to students of statistics and combinatorics, but seems to be unfamiliar to many other people. The formula is easy to use (See §2), easy to derive (See §3), yet is powerful and broadly applicable (See §4). Students who like to see the immediate usefulness of what they are learning find it a most interesting topic. It is hoped that this paper can serve as a basis for a few simple classroom lessons on Newton's Formula.

\section{EXTENDING A SEQUENCE}

Consider the sequence

(A) $5,5,7,12,24,50,100,187, \ldots$

As everyone knows the following question is meaningless.

(Q1) What number follows 187 in sequence (A)?

After all a sequence is a function $f$ from the set of whole numbers into the set of real numbers, and the mere fact that the values $f(0)$ through $f(7)$ are known

$$
\begin{gathered}
f(0)=5, f(1)=5, f(2)=7, f(3)=12, \\
f(4)=24, f(5)=50, f(6)=100, f(7)=187
\end{gathered}
$$

tells us nothing about $f(8)$. Any real number at all could be assigned to 8 by $f$. There are infinitely many sequences which begin with the numbers in (A).

But it would be discriminatory to simply brush off (Q1) as meaningless when we admit (perhaps grudgingly) that we really know what is intended by the technically ambiguous ellipsis marks in (B) and (C) below.

(B) $1,3,5,7,9, \ldots$

(C) $1,2,4,7,11, \ldots$

When we look at (B) we see immediately a simple pattern in the given terms of the sequence: each term is two more than the preceding term, Or, putting it differently, the "sequence of first differences" is all 2's and hence the "sequence of second differences" is identically zero.

$$
\begin{array}{lllllll}
\text { sequence } & 1 & 3 & 5 & 7 & 9 & \ldots
\end{array}
$$




$\begin{array}{ccccc}\text { first differences } & 2 & 2 & 2 & 2\end{array} \quad \begin{array}{llll}\ldots \\ \text { second differences }\end{array} \quad \begin{array}{llll}0 & 0 & 0 & \ldots\end{array}$

Thus, even though incomplete sequence (B) is ambiguous ("I am

again listing successive difference sequences makes one stand out clearly

$\begin{array}{cccccc}\text { sequence } & 1 & 2 & 4 & 7 & 11 \\ \text { first differences } & 1 & 2 & 3 & 4 & \ldots \\ \text { second differences } & 1 & 1 & 1 & \ldots \\ \text { third differences } & & 0 & 0 & \ldots & \end{array}$

We suppose that the writer of (C) had in mind "the function $f: W$ $\rightarrow R$ such that $f(0)=1, f(1)=2, f(2)=4$, and such that the sequence of third differences is the zero sequence." On the basis of this supposition alone we can easily extend the incomplete sequence (C) by filling in the blanks in the alphabetical order indicated below. (Try it.)

$$
\begin{aligned}
& \text { sequence } 124 \text { f } 12 \text { (I).. } \\
& \text { first differences } \quad a[b][a][a] \text {.. } \\
& \text { second differences } c][\mathbb{g}][] \text {.. } \\
& \text { third differences } \quad \begin{array}{lllll}
0 & 0 & 0 & \ldots
\end{array}
\end{aligned}
$$

Consider now the original sequence (A). No pattern is immediately obvious, but writing out successive difference sequences soon reveals one.

$$
\begin{aligned}
& \begin{array}{lllllllll}
5 & 5 & 7 & 12 & 24 & 50 & 100 & 187 & \ldots
\end{array} \\
& \begin{array}{llllllll}
0 & 2 & 5 & 12 & 26 & 50 & 87 & \ldots
\end{array} \\
& \begin{array}{llllllll}
2 & 3 & 7 & 14 & 24 & 37 & \ldots
\end{array} \\
& \begin{array}{llllll}
1 & 4 & 7 & 10 & 13 & \ldots
\end{array} \\
& \begin{array}{lllll}
3 & 3 & 3 & 3 & \ldots
\end{array} \\
& \begin{array}{llll}
0 & 0 & 0 & \ldots
\end{array}
\end{aligned}
$$


It is "natural" to supply (A) with the hypothesis, "the sequence of fifth differences is zero." On the basis of this assumption we answer the original question (Q1)—What number follows 187?-by tacking a fourth zero on the sequence of fifth differences and deducing that successive entries up and to the right are 3,16, 53, 140, 327. (Check this.) The number following 187 in sequence (A) is 327 just as surely as the number following 9 in sequence (B) is 11 .

Consider now a second question.

(Q2) What is the 101th term in sequence (A)?

Again strictly speaking this is a meaningless question. But if we supply the additional (natural) condition that all fifth differences be zero, then the question cannot be ducked. We know that under this hypothesis there is just one correct answer. In fact, we know in principle how to find it. We need only continue appending zeros to the incomplete sequence of fifth differences and filling in entries from the bottom up until we have 101 terms in the original sequence. In practice, however, this would be an enormous job. What we really need is an explicit formula for $f(n)$ into which we can plug 100 for $n$.

Fortunately there is a very easy procedure, Newton's Method, for writing down such a formula. One simply uses the leading terms from each of the non-zero sequences in the difference sequence analysis (4) as coefficients on the binomial coefficients $\left(\begin{array}{l}n \\ 0\end{array}\right),\left(\begin{array}{l}n \\ 1\end{array}\right),\left(\begin{array}{l}n \\ 2\end{array}\right)$, $\ldots\left[\right.$ Recall that $\left(\begin{array}{l}n \\ 0\end{array}\right)=1 ;\left(\begin{array}{l}n \\ r\end{array}\right)=\frac{n(n-1 \ldots(n-r+1)}{r !}$ for 1 $\leq r \leq n ;\left(\begin{array}{l}n \\ r\end{array}\right)=0$ if $\left.r>n.\right]$ The formula for the sequence (A), for example, is

$$
f(n)=5\left(\begin{array}{l}
n \\
0
\end{array}\right)+0\left(\begin{array}{l}
n \\
1
\end{array}\right)+2\left(\begin{array}{l}
n \\
2
\end{array}\right)+1\left(\begin{array}{l}
n \\
3
\end{array}\right)+3\left(\begin{array}{l}
n \\
4
\end{array}\right)
$$

In particular

$$
\begin{gathered}
f(100)=5\left(\begin{array}{c}
100 \\
0
\end{array}\right)+0\left(\begin{array}{c}
100 \\
1
\end{array}\right)+2\left(\begin{array}{c}
100 \\
2
\end{array}\right)+1\left(\begin{array}{c}
100 \\
3
\end{array}\right)+3\left(\begin{array}{c}
100 \\
4
\end{array}\right) \\
=5+0+\frac{2 \cdot 100 \cdot 99}{2 \cdot 1}+\frac{1 \cdot 100 \cdot 99 \cdot 98}{3 \cdot 2 \cdot 1}+\frac{3 \cdot 100 \cdot 99 \cdot 98 \cdot 97}{4 \cdot 3 \cdot 2 \cdot 1} \\
=11,935,280
\end{gathered}
$$

The 101th term of sequence (A) is $11,935,280$. 
For sequence (B) the general formula is obtained by using the leading entries of the non-zero sequences in (1). That is

$$
f(n)=1\left(\begin{array}{l}
n \\
0
\end{array}\right)+2\left(\begin{array}{l}
n \\
1
\end{array}\right)
$$

which can be written in usual polynomial notation as

$$
f(n)-2 n+1
$$

For sequence (C)-see (2)-the general formula is

$$
f(n)=1\left(\begin{array}{l}
n \\
0
\end{array}\right)+1\left(\begin{array}{l}
n \\
1
\end{array}\right)+1\left(\begin{array}{l}
n \\
2
\end{array}\right)
$$

which in usual polynomial notation becomes

$$
\begin{aligned}
f(n) & =1 \cdot 1+1 \cdot n+\frac{n \cdot(n-1)}{2} \\
& =1 / 2 n^{2}+1 / 2 n+1
\end{aligned}
$$

Derivation of Newton's Formula

Suppose $f$ is a sequence; that is $f$ is a function from $W$ into $R$. Define a new sequence $\Delta f$, called the sequence of first differences of $f$, as follows

$$
\Delta f(n)=f(n+1)-f(n) \quad \forall n \in W
$$

The sequence of first differences of $\Delta f$ is written $\Delta^{2} f$ and is called the sequence of second differences of $f$. Thus

$$
\Delta^{2} f(n)=f(n+1)-\Delta f(n) \quad \forall n \in W
$$

Sequences of third, fourth, ... differences are defined in the same way. In general the sequence of $k$ th differences of $f$, written $\Delta^{k} f$, is defined recursively by

$$
\Delta^{k} f(n)=\Delta^{k-1} f(n+1)-\Delta^{k-1} f(n) \quad \forall n \in W
$$

Newton's Theorem

If $f$ is a sequence whose sequence of $(m+1)$ th differences is the zero sequence then $f$ is given by the formula

$$
f(n)=f(0)\left(\begin{array}{l}
n \\
0
\end{array}\right)+\Delta f(0)\left(\begin{array}{l}
n \\
1
\end{array}\right)
$$




$$
+\Delta^{2} f(0)\left(\begin{array}{l}
n \\
2
\end{array}\right)+\ldots+\Delta^{m} f(0)\left(\begin{array}{c}
n \\
m
\end{array}\right)
$$

Proof

Consider the array consisting of the original sequence and its successive difference sequences

$$
\begin{array}{cccccc}
f(0) & f(1) & f(2) & f(3) & f(4) & f(5) \ldots \\
\Delta f(0) & \Delta f(1) & \Delta f(2) & \Delta f(3) & \Delta f(4) \ldots \\
\Delta^{2} f(0) & \Delta^{2} f(1) & \Delta^{2} f(2) & \Delta^{2} f A 3 a & \ldots \\
\Delta^{3} f(0) & \Delta^{3} f(1) & \Delta^{3} f(2) & \ldots \\
\Delta^{4} f(0) & \Delta^{4} f(1) & \ldots
\end{array}
$$

Each entry in the top row can be expressed in terms of the entries along the left edge. Trivially

$$
f(0)=f(0)
$$

and of course $f(1)-f(0)=\Delta f(0)$ implies

$$
f(1)=f(0)+\Delta f(0)
$$

Replacing $f$ by $\Delta f$ in (8)-i.e. ignoring the top row in (6) for a moment—yields

$$
\Delta f(1)=\Delta f(0)+\Delta^{2} f(0)
$$

Now $f(2)-f(1)=\Delta f(1)$ implies $f(2)=f(1)+\Delta f(1)$ which, in view of $(8)$ and $\left(8^{\prime}\right)$, becomes

$$
f(2)=f(0)+2 \Delta f(0)+\Delta^{2} f(0)
$$

Replacing $f$ by $\Delta f$ in (9) yields

$$
\Delta f(2)=\Delta f(0)+2 \Delta^{2} f(0)+\Delta^{3} f(0)
$$

Substituting from (9) and $\left(9^{\prime}\right)$ into the equation $f(3)=f(2)+\Delta f(2)$ yields

$$
f(3)=f(0)+3 \Delta f(0)+3 \Delta^{2} f(0)+\Delta^{3} f(0)
$$

and so forth.

The general formula 


$$
\begin{array}{r}
f(r)=\left(\begin{array}{l}
r \\
0
\end{array}\right) f(0)+\left(\begin{array}{l}
r \\
1
\end{array}\right) \Delta f(0) \\
+\left(\begin{array}{l}
r \\
2
\end{array}\right) \Delta^{2} f(0)+\ldots+\left(\begin{array}{l}
r \\
r
\end{array}\right) \Delta^{r} f(0)
\end{array}
$$

can be established rigorously by a straight forward induction on $r$ using the Pascal Triangle identity

$$
\left(\begin{array}{l}
r \\
p
\end{array}\right)+\left(\begin{array}{c}
r \\
p+1
\end{array}\right)=\left(\begin{array}{c}
r+1 \\
p+1
\end{array}\right)
$$

Consider now the formula (5). We prove that it is satisfied for all $n \in W$ by considering three cases.

Case $1 n=m$. In this case (5) is just (11) with $r=m$.

Case $2 n<m$. Then formula (5) is satisfied in the form

$$
\begin{gathered}
f(n)=\left[f(0)\left(\begin{array}{l}
n \\
0
\end{array}\right)+\Delta f(0)\left(\begin{array}{l}
n \\
1
\end{array}\right)+\ldots+\Delta^{n} f(0)\left(\begin{array}{l}
n \\
n
\end{array}\right)\right]^{+} \\
{\left[\Delta^{n+1} f(0)\left(\begin{array}{c}
n \\
n+1
\end{array}\right)+\ldots+\Delta^{m} f(0)\left(\begin{array}{l}
n \\
m
\end{array}\right)\right]}
\end{gathered}
$$

because the first bracketed quality is $f(n)$ by (11), and the second bracketed quantity is zero since

$$
\left(\begin{array}{l}
n \\
p
\end{array}\right)=0 \text { if } n<p
$$

Case $3 n>m$. Letting $r=n$ in (11) yields

$$
\begin{aligned}
f(n) & =\left[f(0)\left(\begin{array}{l}
n \\
0
\end{array}\right)+\Delta f(0)\left(\begin{array}{l}
n \\
1
\end{array}\right)+\ldots+\Delta^{m} f(0)\left(\begin{array}{l}
n \\
m
\end{array}\right)\right] \\
& +\left[\Delta^{m+1} f(0)\left(\begin{array}{c}
n \\
m+1
\end{array}\right)+\ldots+\Delta^{n} f(0)\left(\begin{array}{l}
n \\
n
\end{array}\right)\right]
\end{aligned}
$$

But the second bracketed quantity is zero since the $(m+1)$ th differences (and hence all higher differences) of $f$ were assumed to be zero. Omitting the second bracketed quantity from (13) leaves (5).

Q.E.D.

The following corollary reminds us of just how familiar the sequences satisfying the hypothesis of Newton's Theorem are. 


\section{Corollary}

The following conditions of a sequence $f$ are equivalent.

(1) $\Delta^{m+1} f$ is the zero sequence but $\Delta^{m} f$ is not.

$$
\begin{gathered}
\text { (2) } f(n)=f(0)\left(\begin{array}{l}
n \\
0
\end{array}\right)+\Delta f(0)\left(\begin{array}{l}
n \\
1
\end{array}\right) \\
+\Delta^{2} f(0)\left(\begin{array}{l}
n \\
2
\end{array}\right)+\ldots+\Delta^{m} f(0)\left(\begin{array}{l}
n \\
m
\end{array}\right) \\
\text { and } \Delta^{m} f(0) \neq 0 .
\end{gathered}
$$

(3) $f$ is a polynomial of degree $m$.

\section{Proof}

$(1)=>(2)$ : This is just Newton's Theorem together with the observation that if $\Delta^{m+1} f$ is the zero sequence and $\Delta^{m} f$ is a non-zero sequence then $\Delta^{m} f$ is a non-zero constant sequence so that, in particular, $\Delta^{m} f(0)$ $\neq 0$.

(2) $=>$ (3): This is a simple consequence of the fact that

$$
\left(\begin{array}{l}
n \\
i
\end{array}\right)=\frac{n(n-1) \ldots(n-i+1)}{i !}
$$

is a polynomial of degree $i$.

(3) $=>$ (1): Letting

$$
f(n)=a_{m} n^{m}+a_{m-1} n^{m-1}+\ldots+a_{0}\left(a_{m} \neq 0\right)
$$

and applying the definition

$$
\Delta f(n)=f(n+1)-f(n)
$$

leads, by straightforward calculation, to the conclusion that

$$
\Delta f(n)=m a_{m} n^{m-1}+\text { a polynomial of degree at most } m-2
$$

That is, the differencing operation $\Delta$ decreases the degree of a polynomial by exactly 1 . Thus if $f$ is a polynomial of degree $m$ then $\Delta^{m} f$ is a polynomial of degree 0 -that is, a non-zero constant sequence-and hence $\Delta^{m+1} f$ is the zero sequence.

Q.E.D. 


\section{SOME APPLiCATions}

A frivolous application of Newton's Method is to making good scores on "intelligence tests." Such tests typically include problems which display the first few terms of a sequence and ask for "the" next term. Often the sequences appear to have an eventual difference sequence of zeros so that Newton's method can be used as we did in $\$ 2$.

More interesting applications arise when the sequences occur in nature. We examine a few such cases below.

Figurate Numbers

Figurate numbers are positive integers suggested by regular polygons. Just as squares suggest the "square numbers" (Fig. 1) so regular pentagons suggest the "pentagonal numbers." Figure 2 depicts the first five pentagonal numbers.

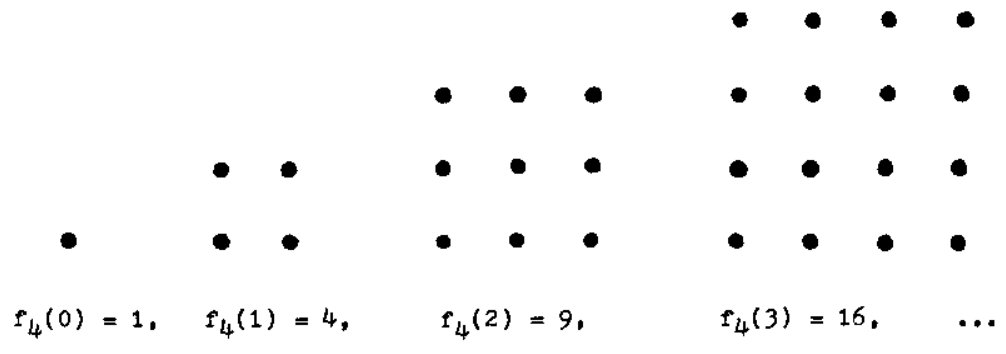

Fig. 1

In general regular p-gons $(p \geq 3)$ give rise to $p$-gonal numbers. We will use Newton's Method to derive the formula for the $n$th p-gonal number.

$$
f_{p}(n-1)=\frac{(p-2) n^{2}-(p-4) n}{2}
$$

Rather than working with the general case right away we begin by considering the special case of pentagonal numbers $(p=5)$.

Running a difference sequence analysis on $f_{5}$ suggests strongly that $\Delta^{3} f_{5}$ is the zero sequence.

$$
\begin{array}{cccccc}
f_{5} & 1 & 5 & 12 & 22 & 35 \ldots \\
\Delta f_{5} & 4 & 7 & 10 & 13 \ldots \\
\Delta^{2} f_{5} & 3 & 3 & 3 \ldots \\
\Delta^{3} f_{5} & 0 & 0 & \ldots
\end{array}
$$




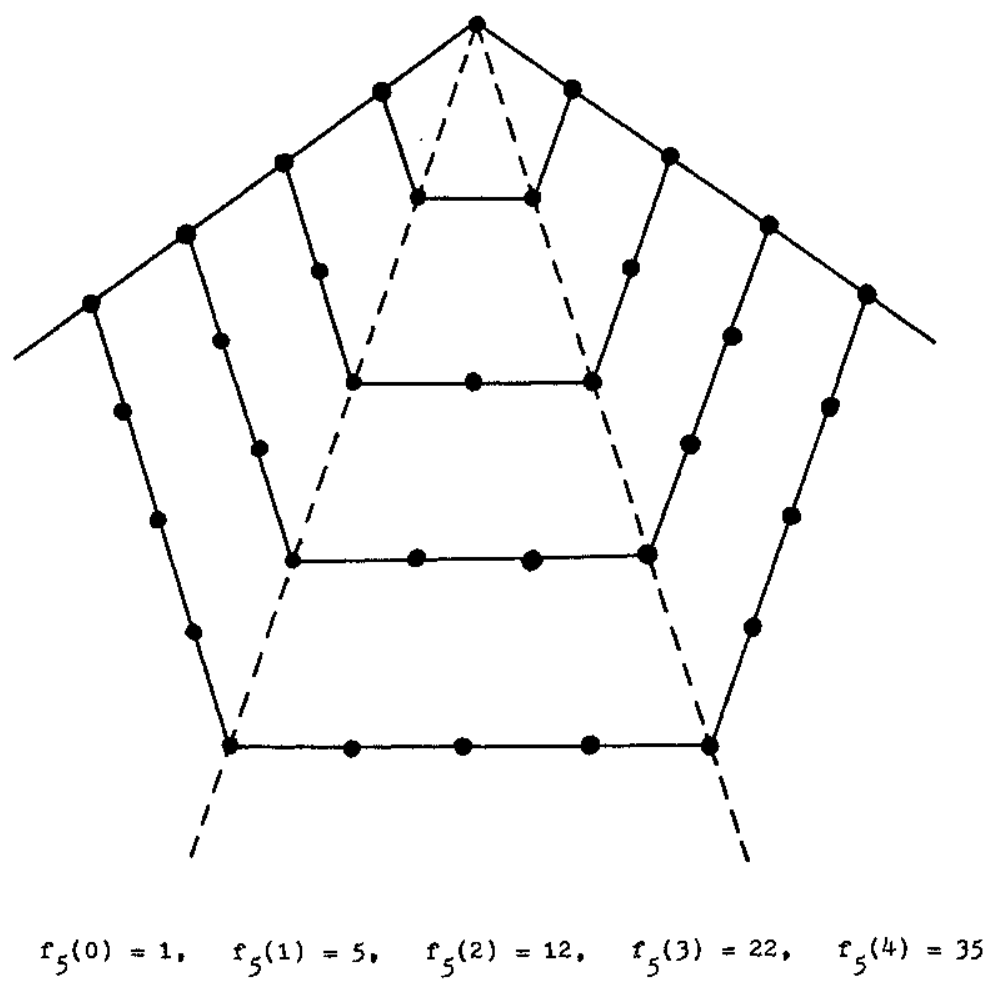

FIG. 2

Thus it appears that Newton's Theorem applies to give this general formula for the $(n+1)$ th pentagonal number:

$$
f_{5}(n)=1\left(\begin{array}{l}
n \\
0
\end{array}\right)+4\left(\begin{array}{l}
n \\
1
\end{array}\right)+3\left(\begin{array}{l}
n \\
2
\end{array}\right)
$$

The question remains: is there geometrical justification (not just arithmetical evidence) for assuming that $\Delta^{3} f_{5}$ is the zero sequence or, what would be as good, that $\Delta^{2} f_{5}$ is the constant sequence 3 , $3,3, \ldots$ ? The answer is yes. Geometrically $\Delta f_{5}(0), \Delta f_{5}(1), \Delta f_{5}(2)$, $\Delta f_{5}(3), \ldots$ represent the number of dots on the first lower border, the second lower border, the third lower border, the fourth lower border, $\ldots$. (See Fig. 3) Geometrically $\Delta^{2} f_{5}(0), \Delta^{2} f_{5}(1), \Delta^{2} f_{5}(2)$, ... represents the gain in dots in moving from each border to the next lower one. This gain is obviously one dot on each of the 3 segments making up a lower border. Thus it is geometrically clear that $\Delta^{2} f_{5}$ is the constant sequence $3,3,3, \ldots$.

In general for regular p-gons it is geometrically clear (See Fig. 4) that

$$
f_{p}(0)=1, f_{p}(1)=p
$$




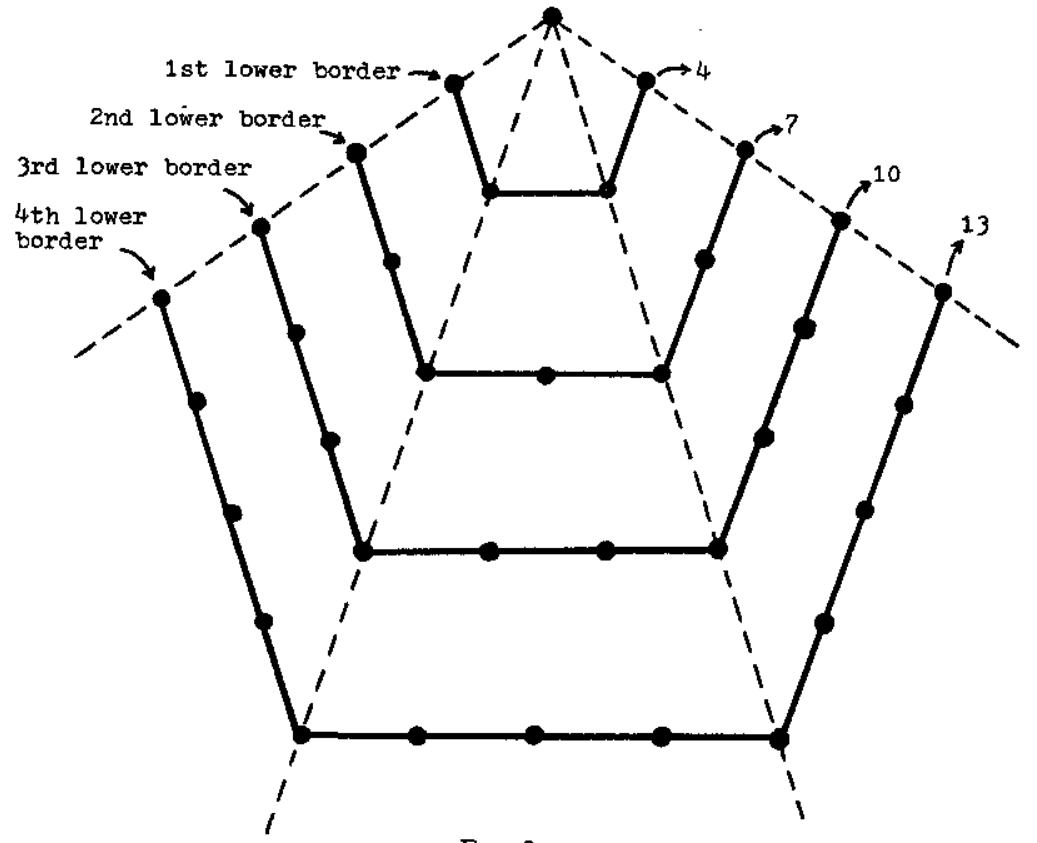

FIG. 3

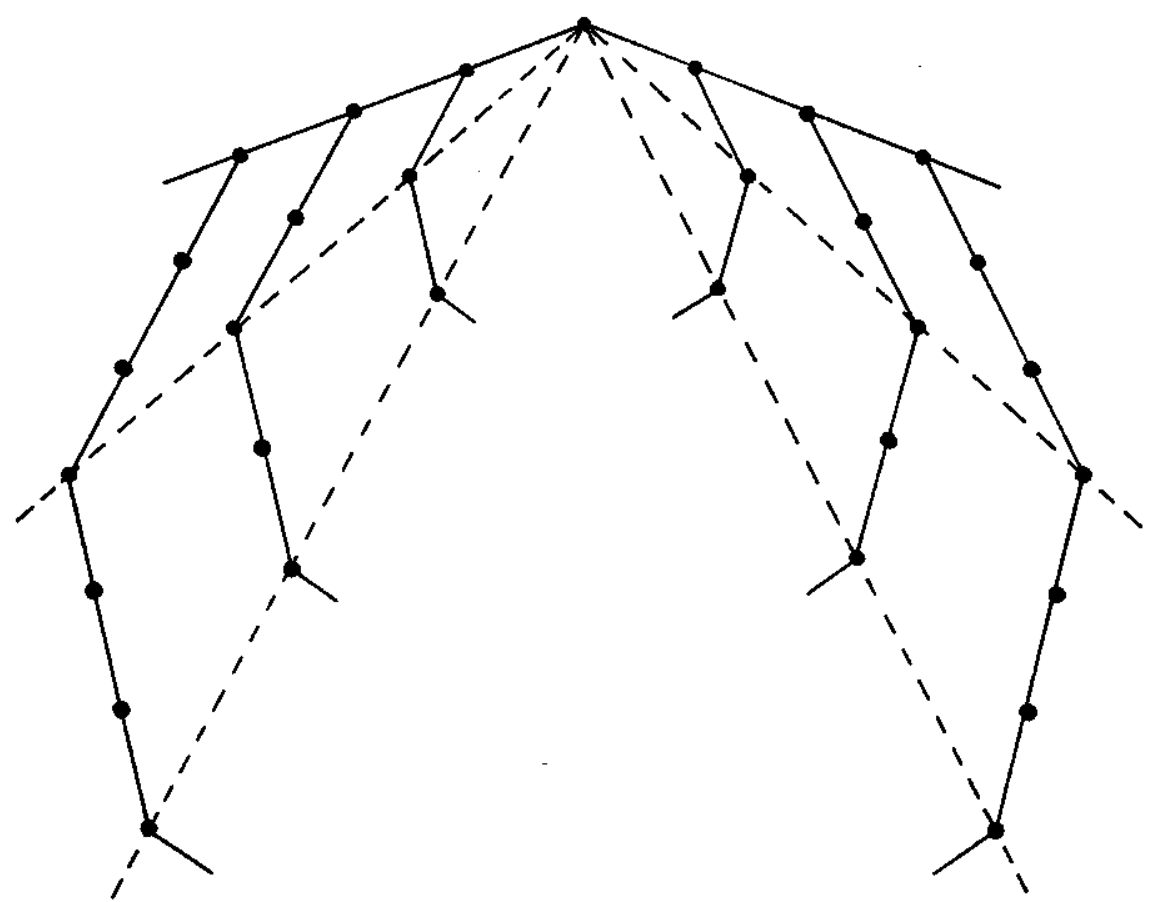

FIG. 4 
and that $\Delta^{2} f_{p}$ is the constant sequence $p-2, p-2, p-2, \ldots$ since each lower border consists of $p-2$ segments. Thus the difference sequence array for $f_{p}$ is

$$
\begin{aligned}
& f_{p} 1 \quad p \square \square \ldots \\
& \begin{array}{lll}
\Delta f_{p} & p-1 \quad \square \square \square \quad \ldots
\end{array} \\
& \Delta^{2} f_{p} \quad p-2 \quad p-2 \quad p-2 \quad p-2 \quad \ldots \\
& \Delta^{3} f_{p} 0000 \ldots
\end{aligned}
$$

from which we conclude, by Newton's Formula that

$$
\begin{aligned}
f_{p}(n)= & 1\left(\begin{array}{l}
n \\
0
\end{array}\right)+[p-1]\left(\frac{n}{061}\right) \\
& +[p-2]\left(\begin{array}{l}
n \\
2
\end{array}\right)
\end{aligned}
$$

Simple algebra shows that formulas (14) and (15) agree.

\section{Polya's Problems}

Another cluster of geometrical problems which yield to Newton's Method is the well-known triple of problem posed by Polya:

(P1) Into how many sections is a line partitioned by $n$ points?

(P2) Into how many regions can a plane be partitioned by $n$ lines?

(P3) Into how many chunks can space be partitioned by $n$ planes?

Let $s(n), r(n), c(n)$ stand for these numbers of sections, regions, and chunks respectively. Then obviously

$$
\begin{array}{cccccc}
s & 1 & 2 & 3 & 4 & 5 \ldots \\
\Delta s & 1 & 1 & 1 & 1 \ldots \\
& & & &
\end{array}
$$

so that

$$
s(n)=1\left(\begin{array}{l}
n \\
0
\end{array}\right)+1\left(\begin{array}{l}
n \\
1
\end{array}\right)
$$

To deduce a formula for $r(n)$, we make two observations. One is trivial

$$
r(0)=1
$$




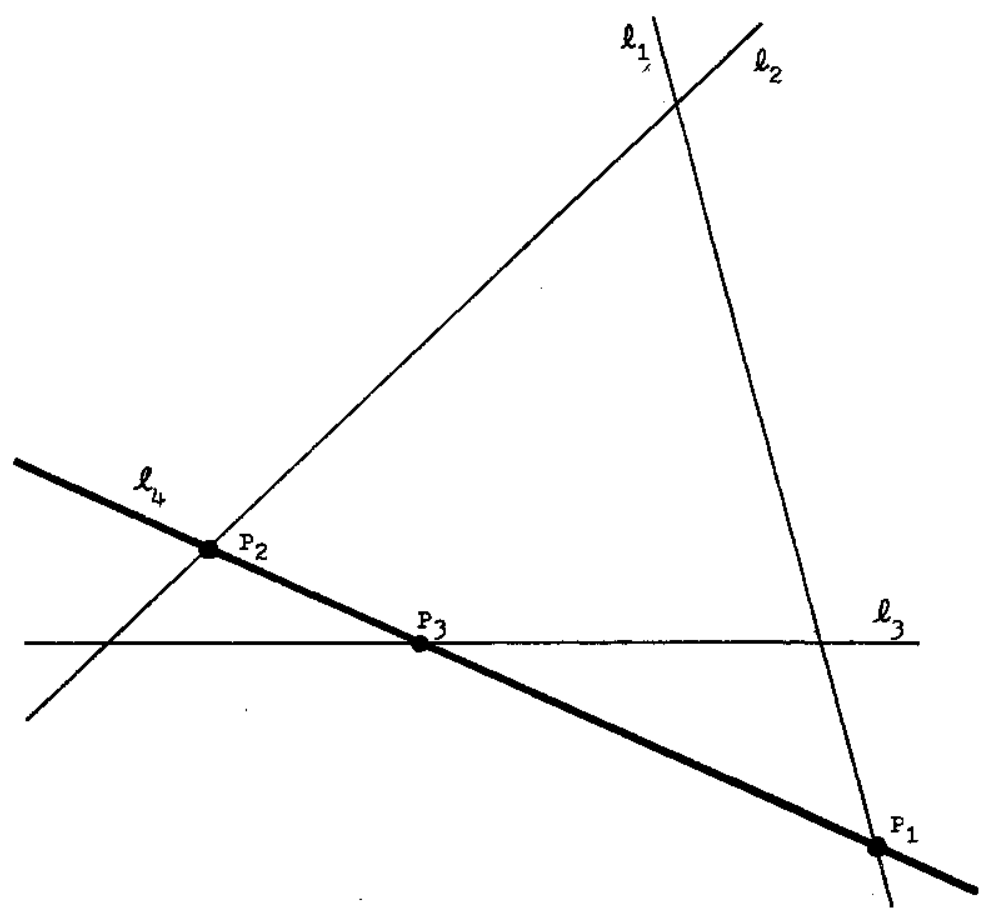

Fig. 5

The other is less obvious

$$
r(n+1)-r(n)=s(n) \text { i.e. } \Delta r=s
$$

Figure 5 illustrates this relationship in the case $n=3$. A plane had been cut up into as many regions as possible $[r(3)=7]$ by 3 lines $-l_{1}$, $l_{2}, l_{3}$. A fourth line $l_{4}$ was then drawn in such a way that no two of the four lines are parallel and no 3 are concurrent. Thus this fourth line meets the previous 3 lines in 3 distinct points $\boldsymbol{P}_{1}, \boldsymbol{P}_{2}$, $P_{3}$. These 3 points partition $l_{4}$ into $s(3)$ sections. Each of these sections divides one of the original $r(3)$ regions into two regions. Thus the gain in regions by drawing the fourth line is $s(3)$. That is,

or

$$
\begin{aligned}
& r(4)=r(3)+s(3) \\
& r(4)-r(3)=s(3)
\end{aligned}
$$

which is (19) in the case $n=3$. Formula (19) can be established in general by paraphrasing the argument just given.

Observations (18) and (19) give us the following information about the sequence $r$. 


$$
\begin{aligned}
& r 1 \square \square \square \quad \ldots \\
& \begin{array}{lllll}
\Delta r & s(0) & s(1) & s(2) & s(3)
\end{array}
\end{aligned}
$$

Combining (20) with (16) tells us that

$$
r(0)=1, \Delta r(0)=1, \Delta^{2} r(0)=1, \Delta^{3} r
$$

is the zero sequence so that, by Newton's Formula,

$$
r(n)=1\left(\begin{array}{l}
n \\
0
\end{array}\right)+1\left(\begin{array}{l}
n \\
1
\end{array}\right)+1\left(\begin{array}{l}
n \\
2
\end{array}\right)
$$

To deduce a formula for $c(n)$ we again need two observations, a trivial one

$$
c(0)=1
$$

and a non-trivial one

$$
c(n+1)-c(n)=r(n) \text { i.e. } \Delta c=r
$$

Figure 6 illustrates this relationship in the case $n=3$. Space had been cup up into as many regions as possible $[c(3)=8]$ by 3 planes $\pi_{1}, \pi_{2}, \pi_{3}$ (which can be thought of as the coordinate planes in Euclidean 3-space). A fourth plane $\pi_{4}$ was then drawn in such a way that it met the previous 3 planes in 3 non-concurrent lines $l_{1}$, $l_{2}, l_{3}$ no 2 of which are parallel. These 3 lines partition $\pi_{4}$ into $r(3)$ regions. Each of these regions acts as a membrane dividing one of the original chunks into two chunks. Thus the gain in chunks by drawing the fourth plane is $r(3)$. That is

$$
c(4)=c(3)+r(3)
$$

or $c(4)-c(3)=r(3)$

which is (24) in the case $n=3$. Formula (24) can be established in general by paraphrasing the argument just given.

Observations (23) and (24) give us the following imformation about the sequence $c$.

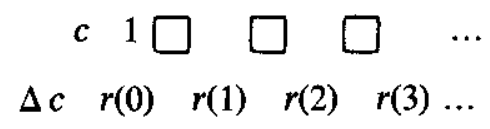

Combining (25) with (20) and (16) tells us that

$$
\begin{gathered}
c(0)=1, \Delta c(0)=1, \Delta^{2} c(0)=1, \Delta^{3} c(0)=1, \\
\Delta^{4} c \text { is the zero sequence }
\end{gathered}
$$




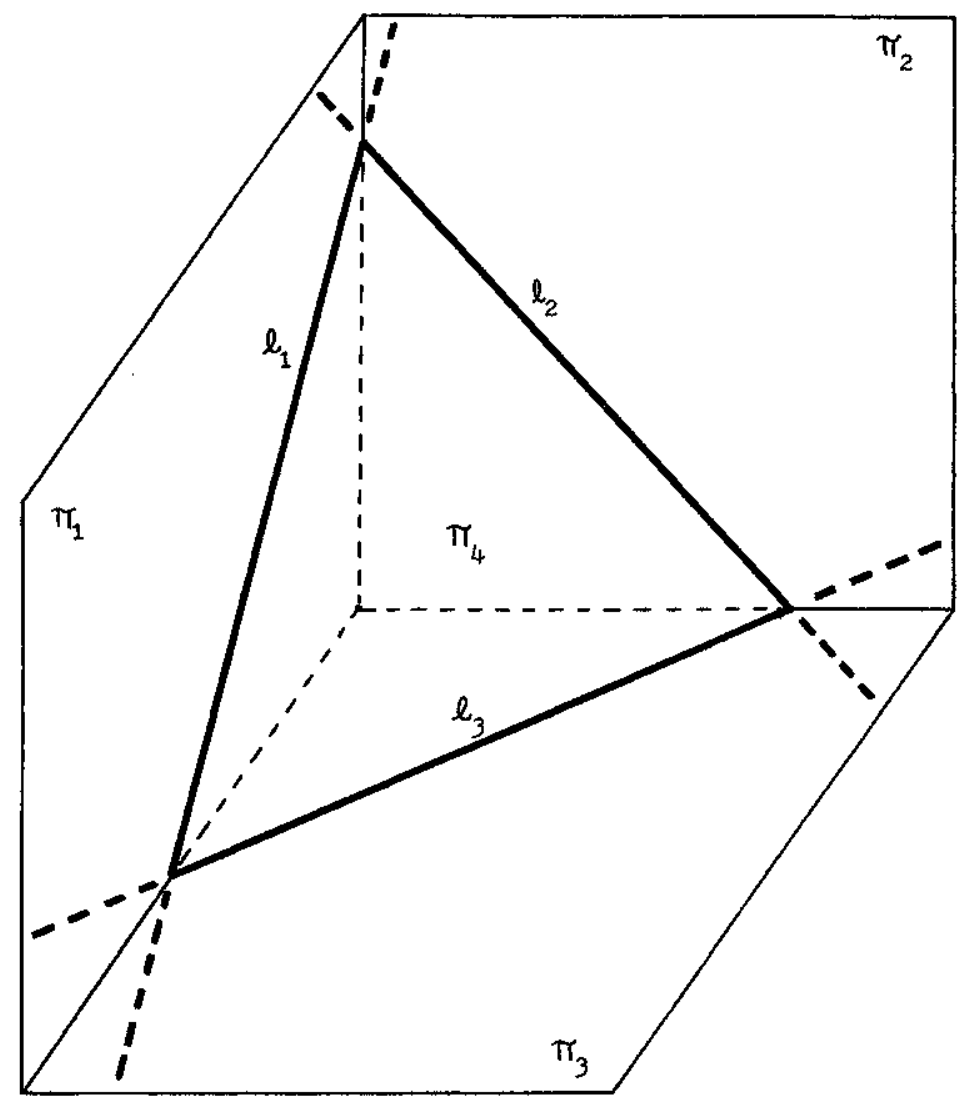

FIG. 6

so that, by Newton's Formula,

$$
c(n)=1\left(\begin{array}{l}
n \\
0
\end{array}\right)+1\left(\begin{array}{l}
n \\
1
\end{array}\right)
$$

$+1\left(\begin{array}{l}n \\ 2\end{array}\right)+1\left(\begin{array}{l}n \\ 3\end{array}\right)$

An Application in Physics

For people who find the previous geometrical "applications" artificial, a whole class of practical, real-world uses of Newton's Formula is suggested by the following. Students in a Physics class have been observing a ball rolling down an inclined plane and have assembled the data in Fig. 7. 


\begin{tabular}{|c|c|}
\hline Time elapsed & Distance traveled \\
in seconds & in meters \\
\hline 0 & 0 \\
1 & .4 \\
2 & 1.6 \\
3 & 3.6 \\
4 & 6.4 \\
\hline
\end{tabular}

FIG. 7

Problem: Write a formula relating distance $d$ (in meters) to time $t$ (in seconds).

Solution: Do a difference analysis on the sequence $d(t)$.

$$
\begin{array}{cccccc}
d & 0 & .4 & 1.6 & 3.6 & 6.4 \\
\Delta d & .4 & 1.2 & 2.0 & 2.8 \\
& \Delta^{2} d & .8 & .8 & .8 \\
& \Delta^{3} & d & 0 & 0 &
\end{array}
$$

The data suggests that $\Delta^{3} d$ is the zero sequence. Thus Newton's Theorem gives

$$
d(t)=0\left(\begin{array}{l}
t \\
0
\end{array}\right)+.4\left(\begin{array}{l}
t \\
1
\end{array}\right)+.8\left(\begin{array}{l}
t \\
2
\end{array}\right)
$$

which becomes

$$
d(t)=.4 t^{2}
$$

in ordinary polynomial notation.

\section{REFERENCES}

JoRdan, Charles. Calculus of Finite Differences. New York: Chelsea, 1965.

POLYA, GEORGE. Induction and Analogy in Mathematics. Princeton: Princeton University Press, 1954.

Willerding, MARGaret. “Figurate Numbers," School Science and Mathematics, LXXII (February 1972), 151-8. 